\title{
Publisher's Note: Self-truncation and scaling in Euler-Voigt- $\alpha$ and related fluid models [Phys. Rev. E 92, 013020 (2015)]
}

\author{
Giuseppe Di Molfetta ${ }^{\circledR}$, Giorgio Krstulovic, and Marc Brachet
}

(C) (Received 26 May 2020; published 3 June 2020)

DOI: 10.1103/PhysRevE.101.069901

This paper was published online on 29 July 2015 with an error in the second author's name. The second author's name should read as "Giorgio Krstulovic." The author's name has been corrected as of 20 May 2020. The author's name is incorrect in the printed version of the journal. 